\title{
Radiation Shielding Issues for Superconducting RF Cavity Test Facility at Fermilab*
}

\author{
I. Rakhno \\ Fermilab, P.O. Box 500, Batavia, IL 60510
}

November 20, 2006

\begin{abstract}
The results of Monte Carlo radiation shielding study performed with the MARS15 code for the final design of the vertical test cryostat facility to be installed in the Industrial Building 1 at Fermilab are presented and discussed.
\end{abstract}

\section{Introduction}

A vertical test cryostat facility for superconducting RF cavities is planned to be installed in the Industrial Building 1 at Fermilab. The operations will be focused on high accelerating gradients-from 20 up to $50 \mathrm{MV} / \mathrm{m}$. In such a case the facility can be a strong radiation source [1]. An initial radiation shielding study for the facility was performed previously [2]. In this study the results of Monte Carlo calculations [3] performed for the final design are described. As far as the groundwater activation with tritium was shown to be negligible [2], emphasis is made on dose distributions around the facility and gamma energy spectra for the final design.

\section{Geometry Model}

A cross section of the developed three-dimensional model of the test facility is shown in Fig. 1. A fragment of the cross section as well as plan view are shown in Fig. 2. As for the color scheme employed to denote materials in the model, the following convention applies to any system: white, light blue, dark green and grey colors correspond to vacuum, air, soil, and regular concrete, respectively. In addition, in this model the violet, red, light green and brown colors correspond to lead, stainless steel, aluminum and borated polyethylene, respectively. The boundaries between different regions are shown with black lines. It

${ }^{*}$ Work supported by the Universities Research Association, Inc., under contract DE-AC02-76CH03000 with the U. S. Department of Energy. 
should be noted also that, when the resolution of a figure is inadequate to show small regions, these regions appear as black ones. In this model the floor level is at $Y=56 \mathrm{~cm}$. The model includes all the components essential from the standpoint of correct description of radiation transport (see also [2]). A real dewar can contain two RF cavities. Only one cavity in its top position is shown in Fig. 1.

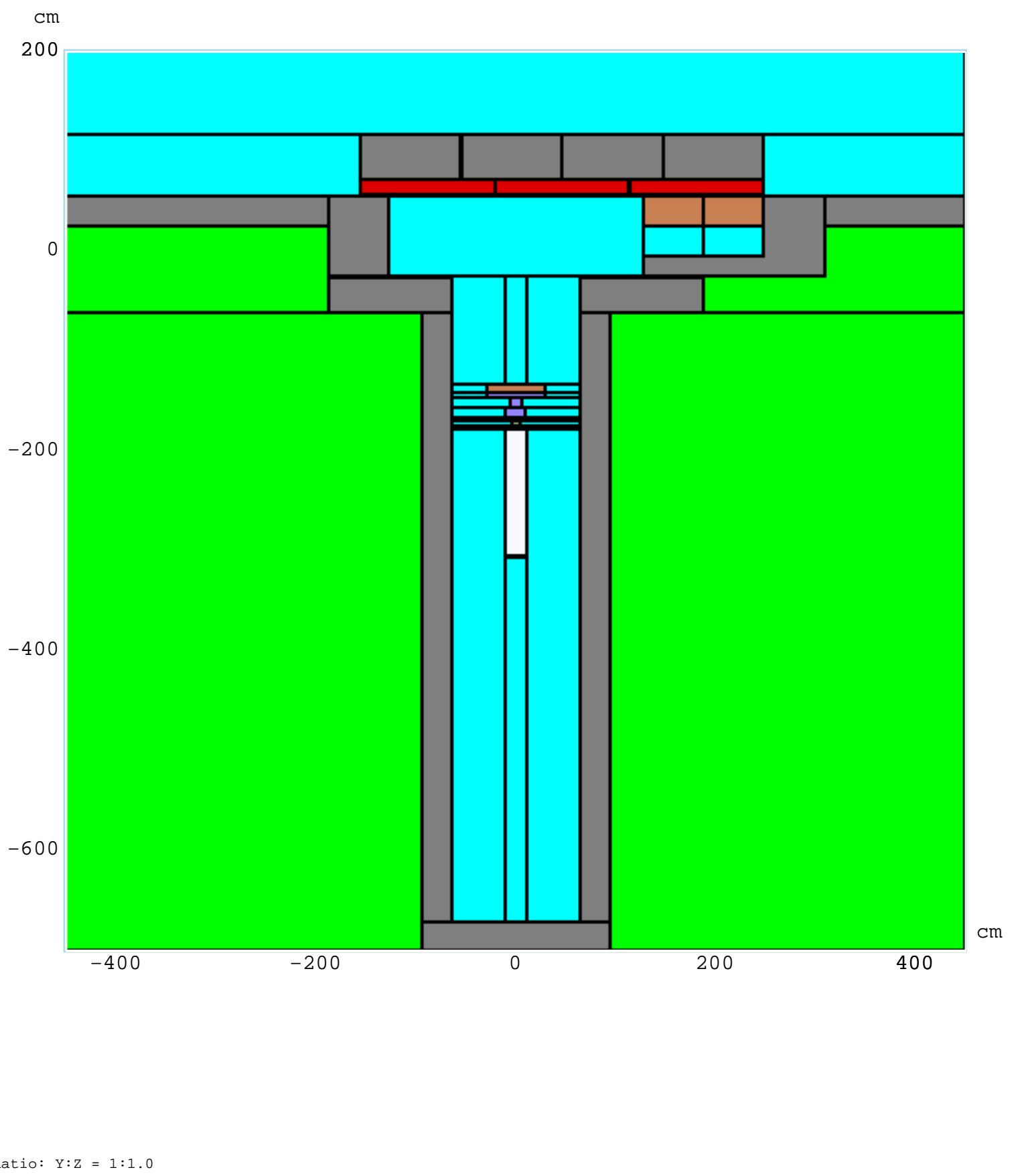

Figure 1: An elevation view of the MARS15 model of the vertical test cryostat facility with an RF cavity in its top position. 


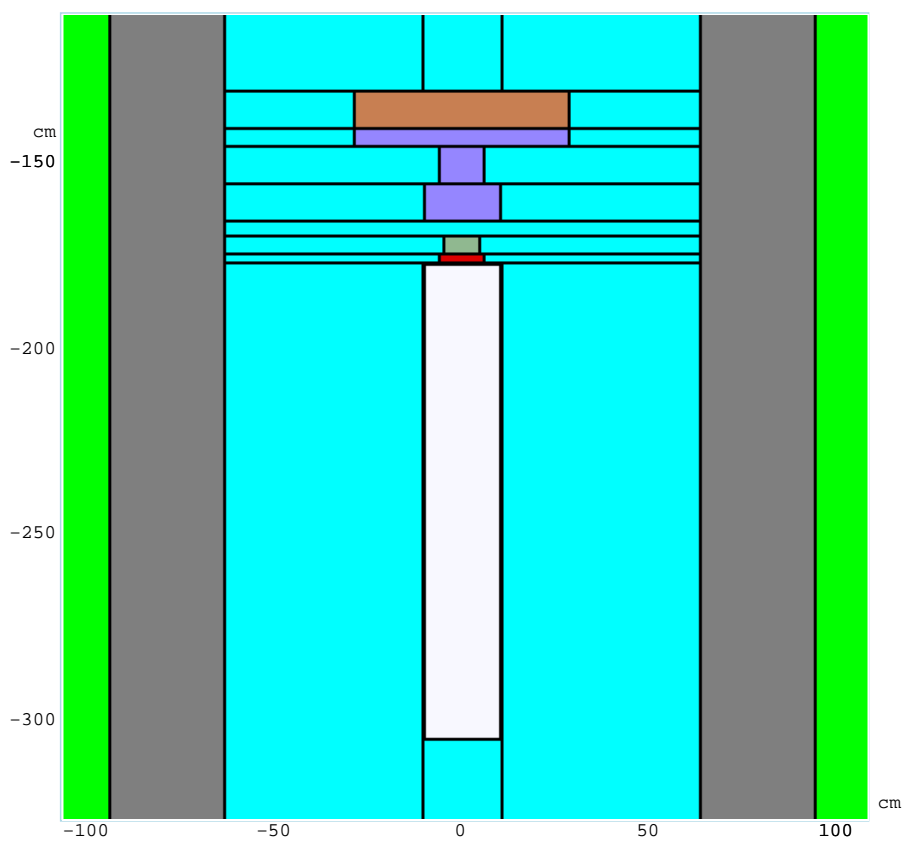

$\stackrel{\mathrm{Y}}{\hookrightarrow} \mathrm{Z}$

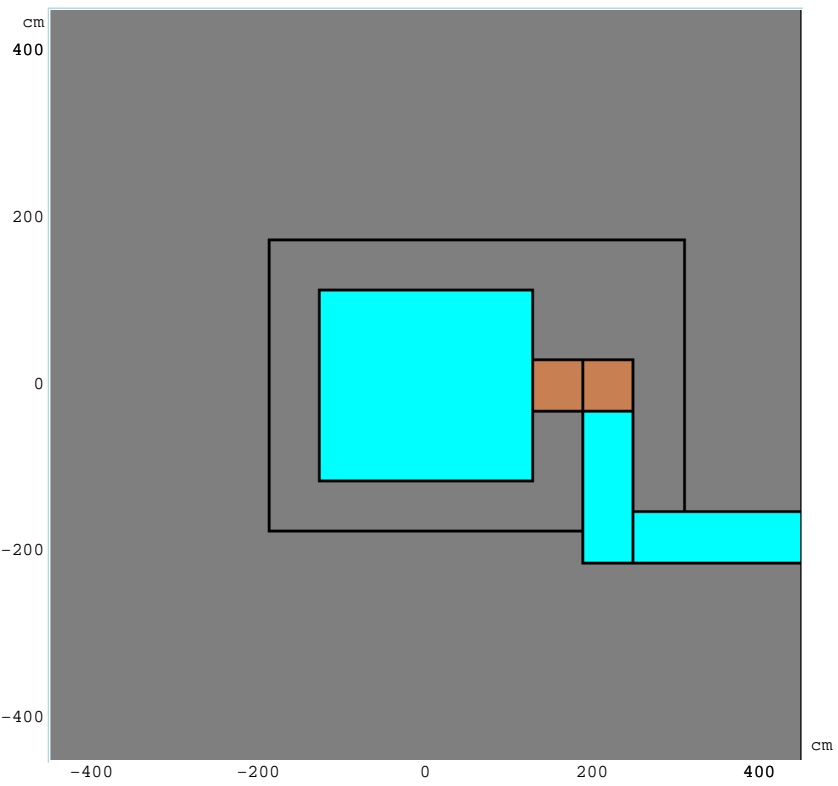

$\stackrel{x}{\longrightarrow} 2$

Figure 2: A fragment of the elevation view of the model (top) showing the details around the RF cavity and fragment of the plan view (bottom) taken at $Y=30 \mathrm{~cm}$ (see Fig. 1). 
In order to describe the final design of the facility, the following updates were applied to the MARS15 model developed previously:

- the length of the shielding along $Z$ axis (see Fig. 1), both steel and concrete, was reduced down to $404 \mathrm{~cm}$ which gives rise to a total weight of the shielding of about 31 tons;

- the radius of the additional shielding layers above the RF cavity (lead and borated polyethylene) was reduced down to $28.7 \mathrm{~cm}$ (see Fig. 2);

- the layout of the instrumentation trench was changed (Fig. 2);

- the amount of concrete in the recessed area was reduced;

- the shielding steel plate is considered to consist of three separate steel plates and the shielding concrete block is considered to consist of four separate concrete blocks;

- gaps were introduced between the concrete floor and the shielding steel plates (10 $\mathrm{mm})$, between the shielding steel plates themselves $(3 \mathrm{~mm})$, and between the shielding concrete blocks $(5 \mathrm{~mm})$.

\section{Source of field-emitted electrons}

Due to lack of a reliable model describing the amount and spatial distribution of the fieldemitted electrons inside the RF cavities, we use two source models to calculate dose distributions around the facility [2].

In the first model we assume that a monodirectional beam of electrons of certain energy $(30,40$, or $50 \mathrm{MeV})$ is going upward and hitting the upper (inner) wall of an RF cavity being tested. Such a scenario implies that the electrons, generated with kinetic energy equal to zero, are accelerated up to the maximum possible energy.

In the second model we assume that the electrons are generated uniformly over the inner surface of the cavity except for its bottom. Angular distribution is assumed to be uniform within a solid angle of $0.293 \pi$ which corresponds to a cone with an opening angle of $45^{\circ}$. For electrons generated on the inner side surface an additional restriction is introduced: their velocity should be directed outside the cavity. Kinetic energy of the electrons, in contrast to the previous study [2], is assumed to depend linearly on their vertical distance from the bottom of the cavity, so that the energy varies from 0 at the bottom up to the nominal value $(30,40$, or $50 \mathrm{MeV})$ on the upper (inner) wall of the cavity. In a sense the second model is more realistic one.

\section{Normalization of calculated data}

The level of the electron current that should be used to normalize the predicted dose and tritium production rate is not known. Twelve years of experimental data from the DESY/TTF 
vertical test facility were analyzed, and are used to make realistic predictions. The x-ray dose rate was measured $5 \mathrm{~cm}$ off axis on top of the stainless-steel top plate (approximately $1 \mathrm{~m}$ to $1.5 \mathrm{~m}$ from the cavity), i.e., between the radiation shielding internal to the dewar and the shielding outside the dewar. An analysis of the maximum x-ray dose rate, at maximum accelerating gradient, showed that the dose rate was less than $5 \mathrm{rem} / \mathrm{hr} 90 \%$ of the time [4]. The maximum x-ray dose rate ever measured was $58 \mathrm{rem} / \mathrm{hr}$. Although $1 \mathrm{~kW}$ is available, the forward power was limited to $250 \mathrm{~W}$ at the cavity for equipment safety reasons. For a single cavity, the $\mathrm{x}$-ray dose rate as a function of accelerating gradient increases approximately exponentially; however, the maximum dose rate varies from cavity to cavity and cannot be correlated directly with accelerating gradient. In addition, it was found that the cavities reaching the highest gradients do not field emit significantly; indeed cavities which put substantial forward power into field emission cannot reach high gradients, so any field-emitted electrons would have lower energy.

Thus, the experimental upper limits are taken independent of the maximum accelerating gradient. The consideration of different gradients is only to show the difference in the secondary particle production for the different electron energies. The maximum gradient achieved was $41 \mathrm{MV} / \mathrm{m}$; a $50 \mathrm{MeV}$ upper limit for electron energy is also very conservative.

The normalization factors were calculated separately for each of the accelerating gradients studied and for each of the two models mentioned in the previous section. In other words, six different normalization factors were detemined.

\section{Results of the calculations}

\subsection{Dose distributions around the facility}

The calculations have been performed for the accelerating gradients of 30,40 , and 50 $\mathrm{MV} / \mathrm{m}$ with the cavity in its top position (see Fig. 1). In contrast to the initial study [2], these calculations were performed with detailed cross sections for generation and transport of low-energy neutrons [5]. The calculated dose distributions around the facility for the gradient of $40 \mathrm{MV} / \mathrm{m}$ are shown in Fig. 3. Table 1 lists the highest dose rates on the top of the movable shielding block. The hottest spots refer to small spatial regions with linear size of about $5 \mathrm{~cm}$ and, therfore, are not quite representative because of inevitable statistical fluctuations even for a long computation time. The doses averaged over spatial regions with linear size of about $30 \mathrm{~cm}$ (which is comparable to linear size of a cross section of a human body) are lower than the data presented in Table 1.

One can see that in the horizontal direction the predicted dose is less than $0.25 \mathrm{mrem} / \mathrm{hr}$ at distances from the $Y$-axis $(Z=0)$ exceeding $300 \mathrm{~cm}$. In the vertical direction a conservative estimate taking into account the dose attenuation in the air [2] enables us to state that the predicted dose is less than $0.25 \mathrm{mrem} / \mathrm{hr}$ at distances from the top of the shielding block that exceed $300 \mathrm{~cm}$. Thus, normal working areas such as offices and workbenches should be located at distances exceeding $300 \mathrm{~cm}$ in order to correspond to the definition of a controlled area of unlimited occupancy [6]. The top of the movable shielding block is an area of minimal occupancy. 


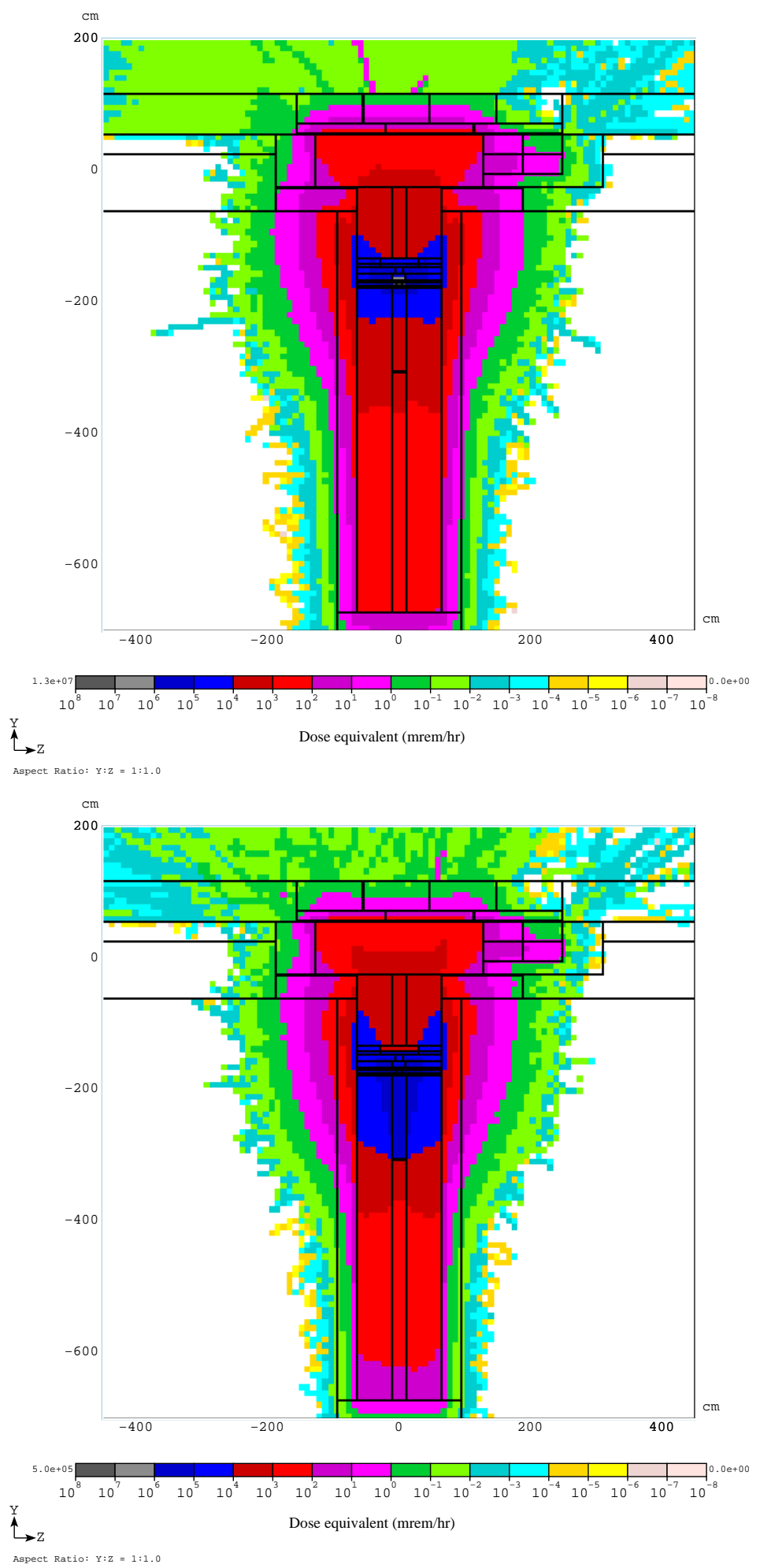

Figure 3: The calculated dose rate distribution around the facility according to the model 1 (top) and model 2 (bottom) (see also section 3) for the accelerating gradient of $40 \mathrm{MV} / \mathrm{m}$ and with the RF cavity in its top position. 
Table 1: Calculated highest dose rate $(\mathrm{mrem} / \mathrm{hr})$ on the top of the removable shielding block for several accelerating gradients $(\mathrm{MV} / \mathrm{m})$ and for the two models described above (see section 3).

\begin{tabular}{|c|c|c|}
\hline $\begin{array}{c}\text { Accelerating } \\
\text { gradient }\end{array}$ & Model 1 & Model 2 \\
\hline 30 & 1.4 & 0.32 \\
40 & 2.5 & 1.4 \\
50 & 2.8 & 1.6 \\
\hline
\end{tabular}

\subsection{Residual activation around the RF cavity}

The distribution of contact residual dose, calculated around the RF cavity for standard conditions (30-days irradiation followed by 1-day cooling), is shown in Fig. 4.

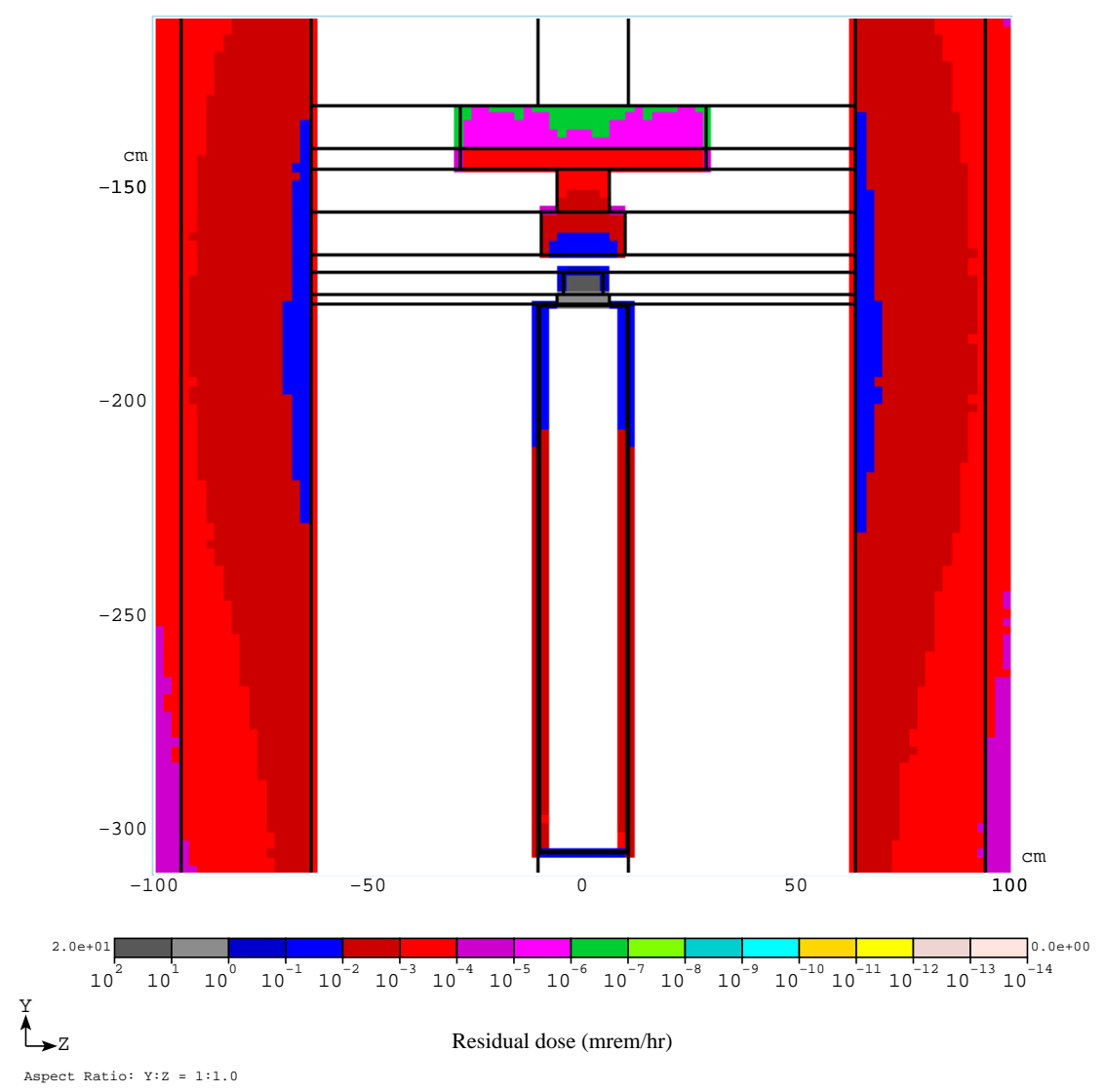

Figure 4: The calculated distribution of contact residual dose around the RF cavity for model 1 (see section 3) and for the accelerating gradient of $50 \mathrm{MV} / \mathrm{m}$ which represents the most conservative estimate of the residual activation. 
The hottest spot—about $20 \mathrm{mrem} / \mathrm{hr}$ - is observed on the aluminum flange. However, in order to get realistic value, one should take into account the following. First, usually a single RF cavity test lasts for about an hour followed by a no less than 18-hour cooling [7]. A detailed calculation for a 1-hour irradiation followed by a 1-day cooling reveals that the residual contact dose for the flange is about $1.2 \mathrm{mrem} / \mathrm{hr}$. Second, for thin and/or small objects such as the flange a scaling factor of about 0.3 should be applied [8]. As a result, the estimated residual contact dose for the flange is about $0.4 \mathrm{mrem} / \mathrm{hr}$ which is much less than the limiting value of $100 \mathrm{mrem} / \mathrm{hr}$ accepted at Fermilab for hands-on maintenance [6].

\subsection{Energy spectrum of gammas}

For diagnostic purposes an X-ray detector should be placed on the top plate, i.e. around $Y=-25 \mathrm{~cm}$. In order to determine the most appropriate type of the detector, energy spectrum of gammas was calculated in a thin layer of the air around this location (see Fig. 5). The predicted dose that a detector can measure in this location is about 1.3 and $1.5 \mathrm{rem} / \mathrm{hr}$ according to the model 1 and model 2, respectively. The doses include, however, a contribution due to neutrons-about $10 \%$.

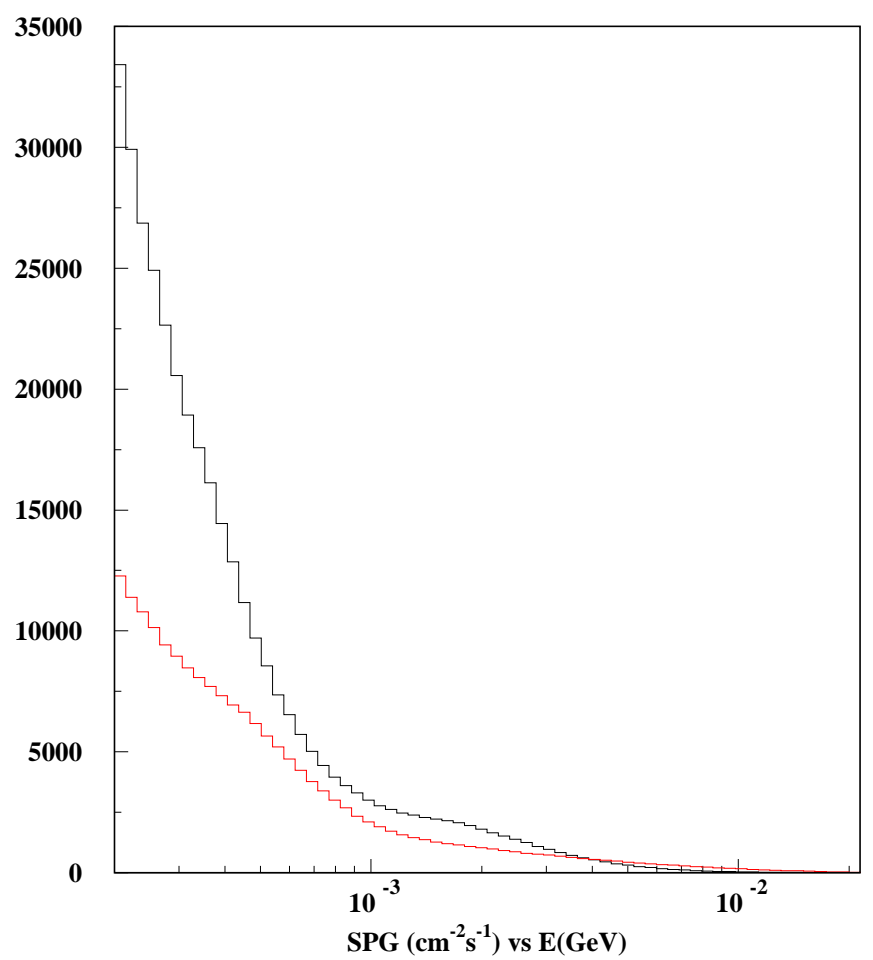

Figure 5: The calculated energy spectrum of gammas in a thin layer of the air around the location $Y=-25 \mathrm{~cm}$ according to model 1 (black line) and model 2 (red line) (see also section 3) for the accelerating gradient of $40 \mathrm{MV} / \mathrm{m}$.

One can see that relative amounts of low- and high-energy gammas in the spectra differ significantly for the two models. For the same model the variation of the spectrum shape 
with accelerating gradient (electron energy) is much less pronounced. At present this uncertainty, as to which spectrum is correct, can not be resolved due to lack of a reliable model describing the amount and spatial distribution of the field-emitted electrons inside the RF cavities.

\section{Dose attenuation in various materials}

It is possible that some minor material replacements will be necessary for technological reasons. In order to estimate the effect of such replacements on the predicted dose, relative dose attenuation in several materials was studied (see Fig. 6). These dependencies can be used, for instance, in order to estimate the amount of lead required to replace the $5-\mathrm{cm}$ aluminum flange (see Fig. 2).
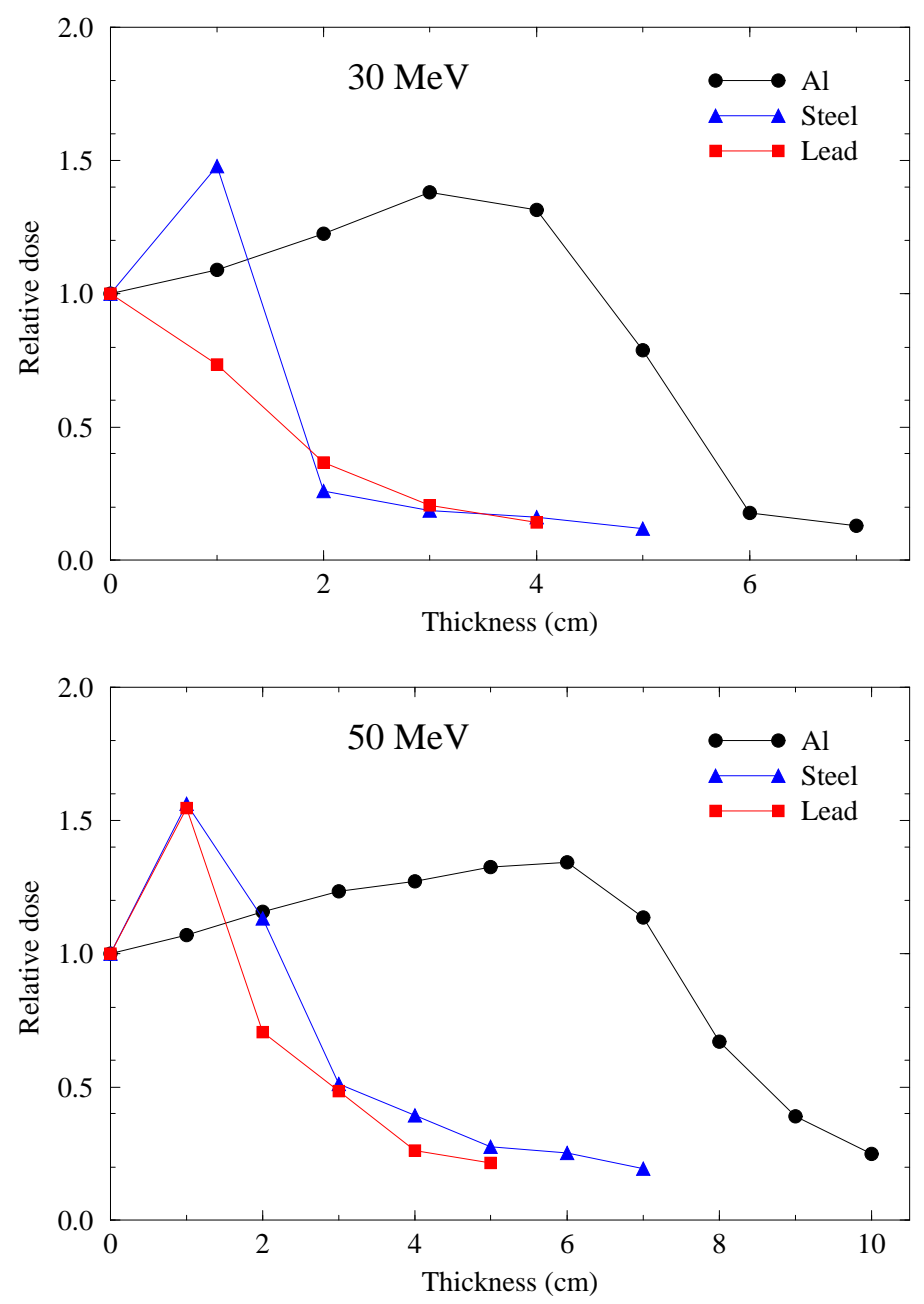

Figure 6: The calculated dose attenuation in layers of several materials $v s$ thickness of the layer for monodirectional and monoenergetic electron beam at normal incidence. The dose in front of the layer due to the incident beam is assumed to be unity. 


\section{Conclusions}

The radiation shielding study was performed with the MARS15 Monte Carlo code for the vertical test cryostat facility. Taking into account all the uncertainties of the employed models as well as the conservative style of our approach, one can state that the suggested shielding will provide for the dose rate in the building not exceeding $5 \mathrm{mrem} / \mathrm{hr}$ in the immediate vicinity of the shielding and not exceeding $0.25 \mathrm{mrem} / \mathrm{hr}$ in normal working areas such as workbenches and offices. According to the Fermilab Radiological Control Manual, this corresponds to the definition of a controlled area of limited and unlimited occupancy, respectively.

\section{Acknowledgements}

The author is grateful to Camille Ginsburg, Joe Ozelis, Valeri Poloubotko, and Cosmore Sylvester of Fermilab for helpful discussions.

\section{References}

[1] J. Knobloch, "Superconducting RF Cavity Test Facilities for Newman LaboratorySafety Report”, Cornell University, Ithaca, NY (2001).

[2] I. Rakhno, "Radiation Shielding Study for Superconducting RF Cavity Test Facility at Fermilab", Fermilab-TM-2350-AD (2006).

[3] N.V. Mokhov et al., "Recent enhancements to the MARS15 code", Fermilab-Conf04/053 (2004); http://www-ap.fnal.gov/MARS/.

[4] W.-D. Moeller, Private communication, DESY (2006).

[5] J. F. Briesmeister, editor, "MCNP - A General Monte Carlo N-Particle Transport Code", Version 4C. Pub. LA-13709-M, Los-Alamos National Laboratory (2000).

[6] "Fermilab Radiological Control Manual", Chapters 2, 3, and 11, http://www-esh.fnal.gov/FRCM/.

[7] J. Ozelis, Private communication, Fermilab (2006).

[8] I. Rakhno, N. Mokhov, A. Elwyn et al., "Benchmarking Residual Dose Rates in a NuMI-like Environment", Fermilab-Conf-01/304-E (2001); N.V. Mokhov, E.I. Rakhno, I.L. Rakhno, "Residual Activation of Thin Accelerator Components", Fermilab-FN-0788-AD (2006). 